\title{
Should Robotics Need Ethical Codes? Robotics and ERP
}

\section{Yu-Cheng Tang*}

\author{
Associate Professor, National Changhua University of Education, Taiwan
}

\author{
Abstract \\ This commentary asks how to provide a role for robotics in automating key transactional Enterprise Resources Planning \\ (ERP) designing processes, and how to integrate ethical considerations into robotic decision making. \\ Keywords \\ Robotics, Enterprise Resources Planning (ERP)
}

\section{Introduction}

The world of manufacturing has changed since Henry Ford first utilized a moving assembly line for the mass production of an entire automobile on December $1^{\text {st }}, 1913$. His innovation reduced the time it took to build a car from more than 12 hours to two hours and 30 minutes. Ford attested that the increased efficiency and reduced labor cost associated with the compartmentalization of production was almost unstoppable. Since that time, manufacturing robots handle a huge variety of practical tasks using much greater speed and efficiency than human workers.

In the Industry 4.0 era, there is heavy use of automation and data exchange in manufacturing environments. The robotics industry has been booming in recent years. Robots can manufacture on a massive scale without the limitations of human tiredness, they can assess production and sales data over time, and communicate in many languages, so companies are using robots in order to optimize productivity and cut their costs.

Apart from the utilization of robots in manufacturing, can robots be utilized in business? Undoubtedly, robots have been applied in many areas of the business environment [1]. For example, robots perform inventory tasks for business within large scale warehouses or sorting facilities. Robots also can communicate with suppliers and customers in a call center role. Above all that, robots also can entertain audiences or customers, e.g., AlphaGo.

\section{When Robots Meet Enterprise Resource Plan- ning (ERP)-Decision Making}

In the business world, Enterprise Resource Planning
(ERP) is the integrated management of core business processes and varied organizational systems and facilitates error-free transactions and production, to enhance an organization's efficiency. Starting in traditional accounting systems, a complex ERP system now covers all business processes of the company.

This commentary asks how to provide a role for robotics in automating key transactional ERP designing processes. Robotics can be combined with highly configurable workflow and data extraction solutions to enable data structure automation, faster approval and exception resolution, and effective management. However robots are not only able to do routine tasks, because their capacity for Artificial Intelligence which mimics human judgements can provide a problem-solving role when unforeseen difficulties arise.

By mimicking human actions at the user interface level and interacting with multiple applications, do robots need to make some decisions for the business? One issue arises when robots start dealing with business matters-does the decision-making need to involve morality codes? When the business scenario is involved, can robots make decisions in accordance with what the owners

*Corresponding author: Yu-Cheng Tang, Associate Professor, National Changhua University of Education, Taiwan, Tel: +886-921327271, Email: loistang888@gmail.com

Received: February 15, 2017; Accepted: April 10, 2017; Published online: April 12, 2017

Citation: Yu-Cheng T (2017) Should Robotics Need Ethical Codes? Robotics and ERP. J Robotics Autom 1(1):22-23

Copyright: @ 2017 Yu-Cheng T. This is an open-access article distributed under the terms of the Creative Commons Attribution License, which permits unrestricted use, distribution, and reproduction in any medium, provided the original author and source are credited. 
want? Even in the early days of robotics, ethical considerations were being stressed, for example in the "Handbook of Robotics, $56^{\text {th }}$ Edition, 2058 A.D." [2], the Three Laws which consider harm and obedience to people:

1. A robot may not injure a human being or, through inaction, allow a human being to come to harm.

2. A robot must obey the orders given it by human beings except where such orders would conflict with the First Law.

3. A robot must protect its own existence as long as such protection does not conflict with the First or Second Laws.

These laws seem to have become embedded in discussions about the design and manufacture of robots. As technology advances quickly, robotics has become a very important part in manufacturing and also in our daily lives. Ethical issues must be considered in parallel to these advances.

Recently, there are a lot of debates about robotics and morality issues as people question whether robots can make the right judgments on morality issues [3-5]. Assessing situations can be complicated by the variation in business ethical codes, local customs and value-systems worldwide. A robot has to identify the business environment, conditions, and possible outcomes for various scenarios. As ethical decision-making in robots becomes more sophisticated, it is necessary to consider how ethi- cal issues are programmed into robots and whether their moral decisions will be trustworthy.

\section{Conclusion}

The robotics world, the business world and the internet of things (IOT) have been merged in our modern world. Everything seems connected with everything else. Robots can do things faster and analyse a variety of applications in one go. However enterprises also need to invest in robust education programs to drive standardization of businesses matters. If standardization is not viable, enterprises must equip robots to collate different data across different modes of situations. The high cost of development in robotics will no doubt be challenged by those who prefer to invest in the human workforce. Above all those, the ethical codes seem to need to be built-in and integrated within the ERP system.

\section{References}

1. Dennis Hartman (2017) What are Robots used for in a business? eHow Contributor.

2. Asimov Isaac (1950) I, Robot.

3. Olivia Goldhill (2016) Coding Ethics, Can we trust robots to make moral decisions? Quartz.

4. Joelle Renstrom (2016) Can Robots make moral decisions? Should they? The Daily Beast.

5. Abishur Prakash (2016) Why robot law around industrial automation varies worldwide. Robotics business review. 